\title{
Review Article \\ Bioinformatics Genes and Pathway Analysis for Chronic Neuropathic Pain after Spinal Cord Injury
}

\author{
Guan Zhang $^{1,2}$ and Ping Yang \\ ${ }^{1}$ Department of Neurobiology, Chongqing Key Laboratory of Neurobiology, Third Military Medical University, \\ Chongqing 400038, China \\ ${ }^{2}$ Cadet Brigade, Third Military Medical University, Chongqing 400038, China
}

Correspondence should be addressed to Ping Yang; cp_yang_1999@yahoo.com

Received 7 April 2017; Revised 9 August 2017; Accepted 7 September 2017; Published 15 October 2017

Academic Editor: Giuseppe Donato

\begin{abstract}
Copyright (c) 2017 Guan Zhang and Ping Yang. This is an open access article distributed under the Creative Commons Attribution License, which permits unrestricted use, distribution, and reproduction in any medium, provided the original work is properly cited.
\end{abstract}

\begin{abstract}
It is well known spinal cord injury (SCI) can cause chronic neuropathic pain (NP); however its underlying molecular mechanisms remain elusive. This study aimed to disclose differentially expressed genes (DEGs) and activated signaling pathways in association with SCI induced chronic NP, in order to identify its diagnostic and therapeutic targets. Microarray dataset GSE5296 has been downloaded from Gene Expression Omnibus (GEO) database. Significant analysis of microarray (SAM), Kyoto Encyclopedia of Genes and Genomes (KEGG) pathway enrichment analysis, and pathway network analysis have been used to compare changes of DEGs and signaling pathways between the SCI and sham-injury group. As a result, DEGs analysis showed there were 592 DEGs with significantly altered expression; among them $\mathrm{Ccl} 3$ expression showed the highest upregulation which implicated its association with SCI induced chronic NP. Moreover, KEGG analysis found 209 pathways changed significantly; among them the most significantly activated one is MAPK signaling pathway, which is in line with KEGG analysis results. Our results show Ccl3 is highly associated with SCI induced chronic NP; as the exosomes with $\mathrm{Ccl} 3$ can be easily and efficiently detected in peripheral blood, Ccl3 may serve as a potential prognostic target for the diagnosis and treatment of SCI induced chronic NP.
\end{abstract}

\section{Introduction}

Neuropathic pain (NP) is a common consequence following spinal cord injury (SCI), which compromises a person's life satisfaction and quality. It is estimated that the mean cost was $\$ 47,518$ for each SCI patient with NP in the USA [1]. According to the statistics of the GoPubMed website (http://www.gopubmed.org/web/gopubmed/), the molecular mechanisms of NP following SCI remain elusive. NP in SCI can be classified as "at-level" pain [2-4], "below-level" pain $[2,3]$, and "above-level" pain [5-7]. According to the International Spinal Cord Injury Pain Classification system [8], pain experienced at or within three dermatomes below the neurologic level of injury is considered at-level neuropathic pain, while pain that is present more than three dermatomes below the level of injury is classified as belowlevel neuropathic pain. While at-level pain results from lesion of nerve roots and/or the spinal cord and is felt at the corresponding segment, below-level pain is a central pain caused by damage to spinal cord pathways, suggesting different pathological mechanisms of pain generation $[9,10]$. However, there is not too much description for above-level neuropathic pain following SCI in previous study. Not rarely, patients suffer both at-level and below-level pain, but at-level pain seems to appear earlier than below-level pain clinically [11].

The prevalence of NP syndromes in the general population is as high as 7 to $8 \%[12,13]$, and approximately $30-50 \%$ of patients with a SCI will develop chronic NP [1416]. And, according to previous studies [17], a total of 140 participants were analyzed, 70 of them were SCI-NP subjects and the remaining 70 controls did not show neuropathic symptoms. SCI can result from trauma, tumor, infection, and degenerative condition; among them the traumatic SCI plays a pivotal role in inducing chronic NP [18]. There are annually $0.25-0.5$ million SCI cases around the world 
and more than $90 \%$ are due to traumatic injury [19]. SCI results in cell loss, disruption of neural circuitry, and chronic functional impairment [20]; thus patients with chronic NP after SCI may benefit from strategies aiming to promote neurogenesis, neural plasticity, and functional recovery such as human umbilical cord-derived mesenchymal stem cell transplantation [21], astrocyte transplantation [22-25], and neural stem cell transplantation $[26,27]$. The mechanisms underlying SCI induced chronic NP remain elusive, and recent advance in neuroscience has implicated that SCI is a polygenic disease and its pathogenic mechanism is associated with changes of gene expression; therefore identification of related genes in chronic NP after SCI could provide new insights into gene function as well as potential diagnostic and therapeutic targets. In this study, we used microarray technology to identify differentially expressed genes (DEGs) and activated signaling pathways in association with SCI induced chronic NP in a mouse SCI model.

\section{Material and Methods}

2.1. Data Source. Microarray technology is a widely used high-throughput tool for measuring gene expression [28-30]. Moreover, previous studies have shown that data from DNA microarray analysis can be reliable and useful for identifying novel targets for clinical diagnostic and therapeutic approaches [31]. Thus, we used the microarray expression profiles (GSE5296), which were extracted from the GEO (https://www.ncbi.nlm.nih.gov/geo/) database, to identify DEGS associated with SCI induced chronic NP. A C57BL6 mouse SCI model was used (https://www.ncbi.nlm.nih.gov/ geo/query/acc.cgi?acc=GSE5296). The experimental group $(n=12)$ was subjected to a moderate injury at the T8 spinal cord segment under isoflurane anesthesia. Total RNA was extracted from sections of rostral regions, caudal regions, and lesion centre from T8 spinal cord injury $(0.4 \mathrm{~cm}$ in length each one). The expression of genes was detected at a series of time points: $0.5,4,24,72 \mathrm{~h}$, and 7 and 28 days after injury, respectively. According to clinical circumstance, patients commonly experience NP during the initial 3-6 months and 3-5 years after SCI [2]. Therefore, it must be noted that neuropathic pain did not appear during the time points we studied. The control group is sham-injured $(n=8)$, with laminectomy only. Global changes were evaluated using Affymetrix Mouse Genome 4302.0 arrays. The experiments were performed three times.

Altered DEGs and signal pathways were compared between injury group and sham-injury group in different regions: rostral, lesion centre, and caudal regions and at serial time points. The lesion centre has the most significant alteration in DEGs and signal pathways, and thus the dates in different time point from lesion centre were chosen to perform the next analysis before normalization processing by RMA (robust multichip average).

2.2. Data Preprocessing. The RMA method [32] is for computing an expression measurement with three steps' process: background-correction, normalization, and summary. The method includes a probe-specific background-correction and a probe selection strategy in which a subset of probes with highly correlated intensities across multiple samples is chosen to summarize gene expression.

2.3. Analysis Methods. GCBI platform (https://www.gcbi.com .cn/gclib/html/index) was mainly used in the whole process. Initially, DEGs were significantly identified in the spinal cord total RNA samples from C57BL6 mouse model of contusion injury in comparison with samples from animals of laminectomy only. Significant analysis of microarray (SAM) is used to study DEGs. $P=0.05$ was used as the significance threshold of screening DEGs, and fold change > 2 was used as the threshold to determine the significance of gene expression difference. Cluster analysis based on Pearson correlation calculation was used to ensure that the screened genes perfectly expressed the differences between SCI group and sham-injury group. Furthermore, GO functional [33] and KEGG analysis were performed to identify the altered pathway involved in the SCI. Significantly enriched GO terms and KEGG pathways with Fold Discovery Rate (FDR) < 0.05 and $P<0.05$ were screened out. Finally, pathway net analysis was to explore the relationships among each pathway. What is more, it must be noted that SAM (significant analysis of microarray) is used to study DEGs (differentially expressed genes), while RMA (robust multichip average) is for normalization procession, and their function are totally different.

\section{Results}

3.1. Data Preprocessing from Lesion Centre. The original data were preprocessed by RMA function with the Affymetrix package of $\mathrm{R}$ language [34]. The original CEL files were switched into probe expression measures, and the probelevel data were converted into gene names by an annotation package supported by the GCBI platform. After excluding the influence of background, the signal values of the samples are still high (Figure 1(a)), assuring the reliability of the analysis results. It can be seen that the black lines were almost on the same line (Figure 1(b)), indicating an excellent degree of standardization, which ensure the accuracy of subsequent data processing. The correlations of all samples are basically very strong (Figure 1(c)), providing the basis of the subsequent cross analysis and system analysis. The data preprocessing results showed that the samples were sufficiently, precisely, and stably enough to support the following analysis.

\subsection{Screening of Differentially Expressed Genes from T8 Lesion} Centre (Ccl3 Was the Maximum Changed Gene Expression Profile among the 592 DEGs). DEGs that were significantly differentially expressed were screened out by use of significance analysis of microarrays (SAM) $[35,36]$ in the GCBI platform. $P<0.05$ and fold change $>2$ were used as the threshold of screening differentially expressed genes. When the number of samples becomes large, we implement a standard analysis method for screening difference genes. In fact, we use the two samples' Welch $t$-test (unequal variances) for two groups' difference analysis and use analysis of variance (ANOVA) for multiple groups (groups count no 


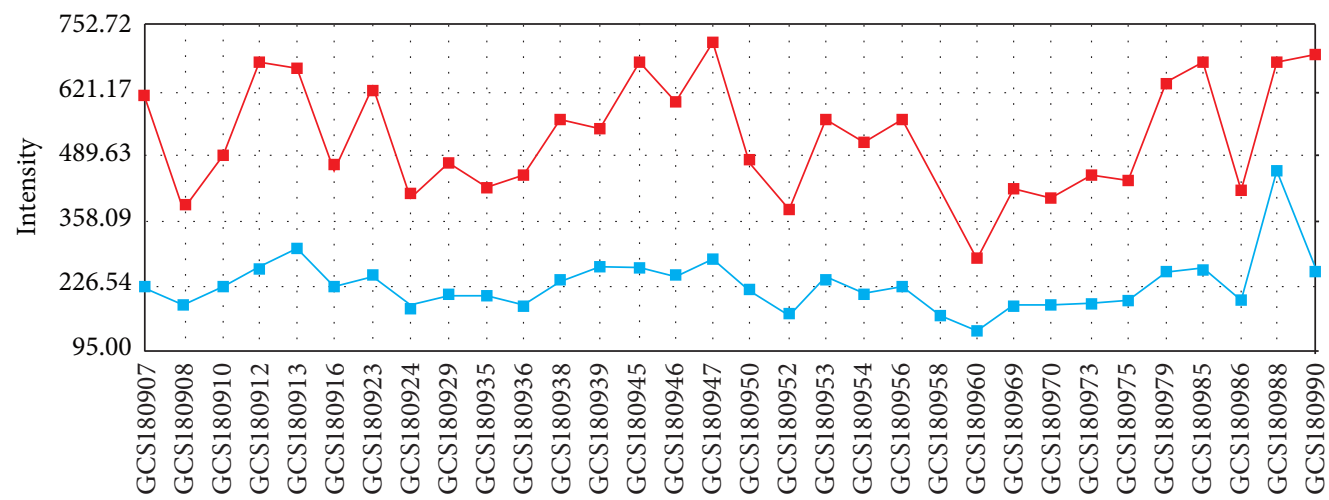

(a)

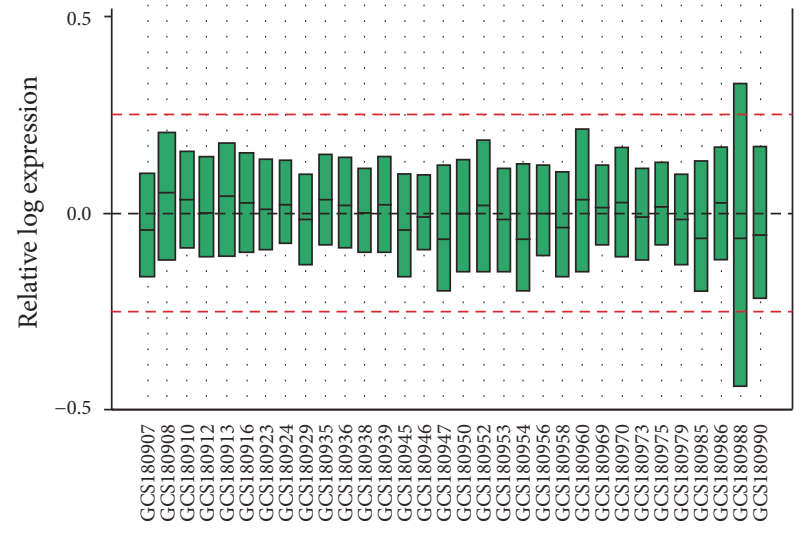

(b)

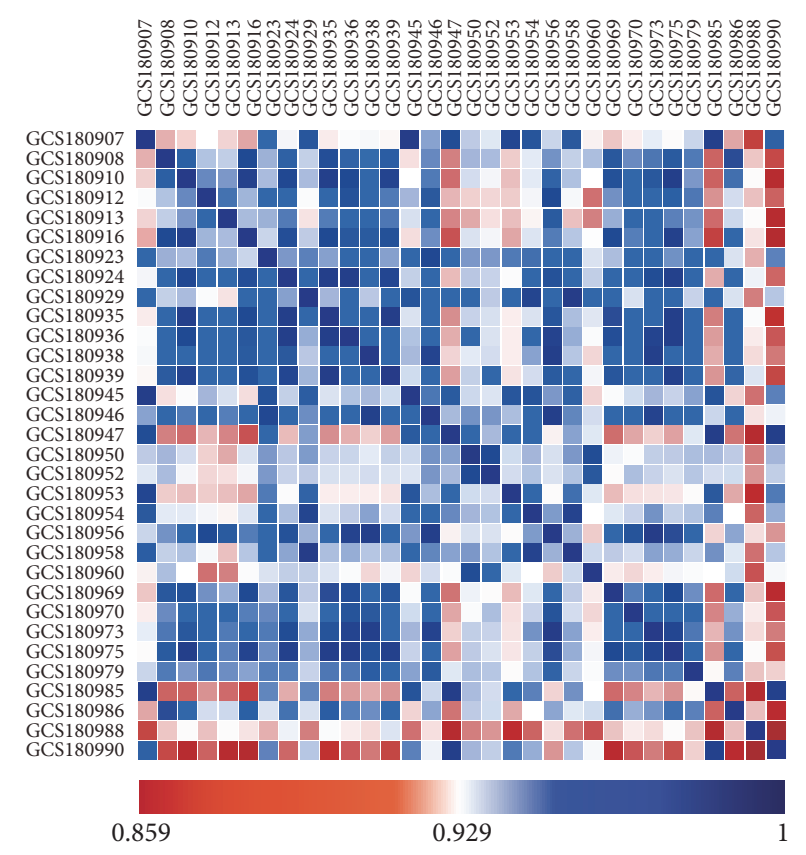

(c)

Figure 1: The background and signal value for each sample. Red represents the average of the values of the sample signals for each sample. Blue represents the average of the background values for each sample. It demonstrated that, after excluding the influence of background, the signal values of the samples are still high (a). The horizontal axis represents the name of samples, while the vertical axis represents the expression value after log conversion. The black lines stand for median, which can be used to identify the degree of standardization after normalization of all samples by the package of R/Bioconductor. It can be seen that the black lines were almost on the same line (b). Sample correlation calculated by injury associated genes expression. Both the horizontal axis and the vertical axis represent the name of samples. The gene expression level from different sample was calculated with Pearson correlation. The closer the point is to the blue color, the greater the correlation is between the two samples. It shows that the correlations of all samples are basically very strong (c).

less than 3). For multiple comparison analysis, we computed the q-value to control the false discovery rate [37]. The top ten largest differences in DEGs were screened with fold change > 2 and $P<0.05$, and the maximum change of gene expression profile was upregulated Ccl3 (Table 1). After heatmap of gene expression differences by gene coexpression network analysis, we found 592 statistically significant DEGs (Figure 2(a)), which is consistent with the known results that SCI is a polygenic disease and its pathogenic mechanism is associated with changes of gene expression. The abscissa value of $\mathrm{Ccl} 3$ is 3.45 (Figure 2(a)), which shows $\mathrm{Ccl} 3$ is the maximum change among all DEGs.
We further calculated the Pearson correlation to construct the distance between the genes and samples and implemented the hierarchical clustering based on used average method for linkage [38]. The top 10 DEGs were listed according to the size of difference and $\mathrm{Ccl} 3$ is the top DEGs which are upregulated. From the horizontal axis at the top, it can be concluded that the samples can be divided into clusters generally: the control group of sham-injury and the experimental group of injury (Figure 2(b)). Moreover, the maximum change of gene expression profile was upregulation of $\mathrm{Ccl} 3$ (fold change $=10.91$, $P=2.20 E-05)$. 


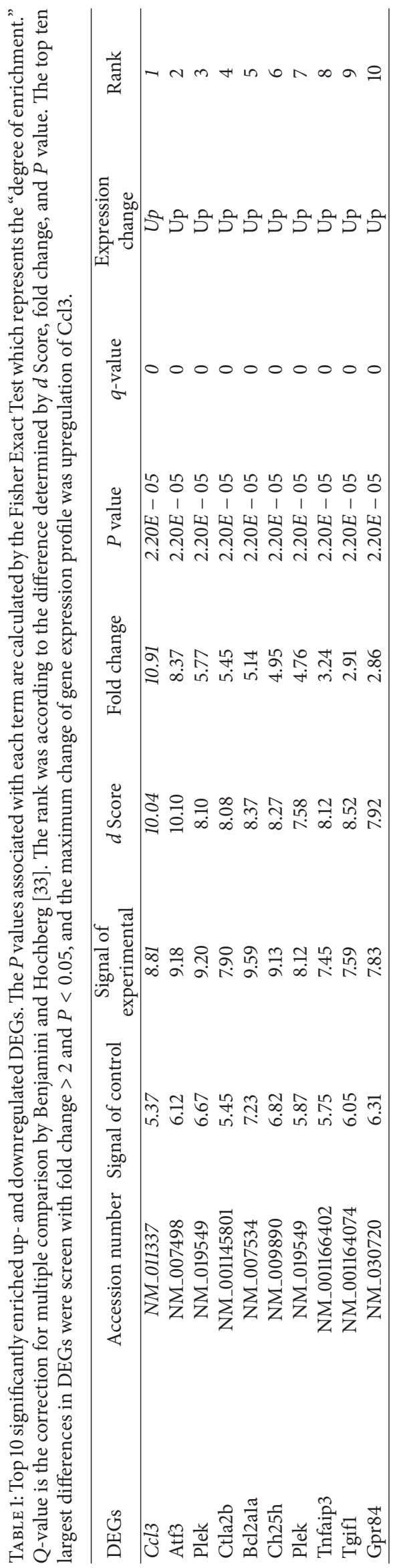




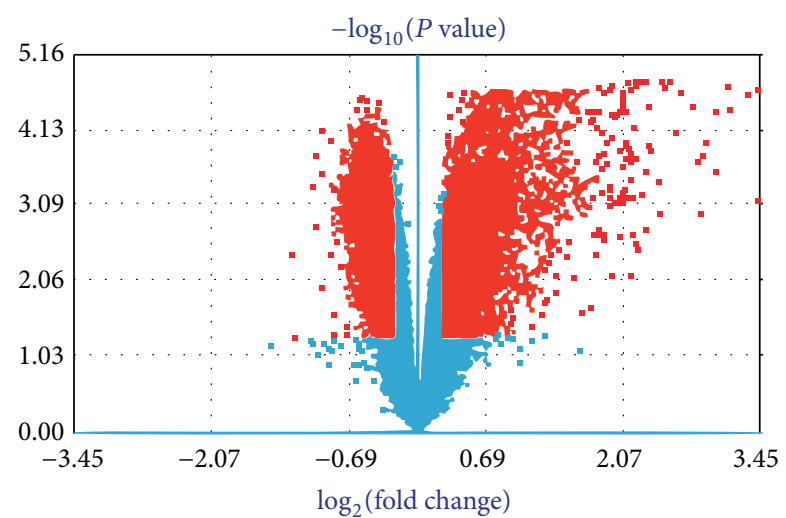

(a)

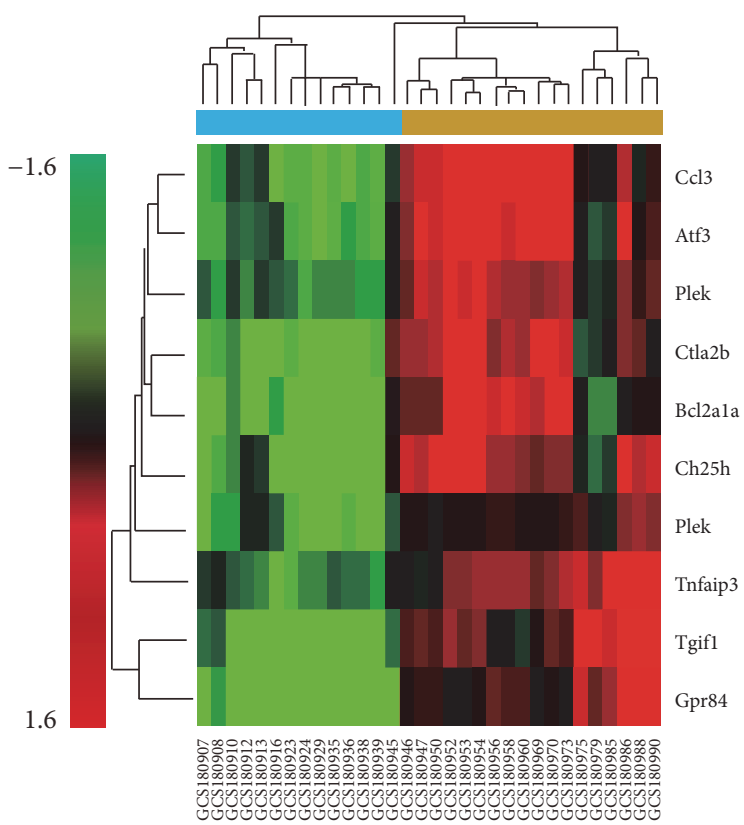

(b)

FIGURE 2: Heatmap of gene expression differences by gene coexpression network analysis. Red dot indicates a differentially expressed gene with statistical significance. Red dots on the right indicate upregulation of gene expression, whereas red dots on the left indicate downregulation of gene expression. Blue indicates that there is no statistically significant difference in gene expression. The greater the ordinate value corresponding to the point is, the greater the difference in gene expression corresponding to that point is. Similarly, the greater the absolute value of the abscissa corresponding to the point is, the greater the difference in gene expression corresponding to that point is. Note that there are 592 statistically significant DEGs. The abscissa value of Ccl3 is 3.45, which means Ccl3 is the maximum change among all DEGs (a). Hierarchical clustering dendrogram of gene expression: the horizontal axis at the bottom represents the name of samples and the vertical axis on the left side represents the degree of gene clustering. The vertical axis on the right side represents the name of genes and the horizontal axis at the top represents the degree of clustering of samples. The red color stands for upregulated while the green color stands for downregulated. The darker red indicates a stronger upregulation in expression and the darker green indicates a stronger downregulation in expression. It can be concluded that the samples can be divided into clusters generally: the control group of sham-injury and the experimental group of injury (b). Moreover, the maximum change of gene expression profile was upregulation of $\mathrm{Ccl} 3$ (fold change $=10.91, P=2.20 E-05)(b)$.

3.3. KEGG Pathway Enrichment Analysis from T8 Lesion Centre (MAPK Signaling Pathway Was the Most Important among the 209 Pathways). Significantly enriched GO terms and KEGG pathways with FDR $<0.05$ were screened out. The rank was according to the enrichment score, $P$ value, and FDR. KEGG biological pathway enrichment analysis found that MAPK signaling pathway (enrichment score $=5.68, P=$ $3.38 E-74$, and FDR $=8.78 E-72$ ) was the most important one among the 209 pathways according to the enrichment scores (Table 2).

\subsection{Pathway Network Analyses from T8 Lesion Centre (MAPK} Signaling Pathway Was Also the Most Important Pathway). The interaction in KEGG was used to construct the interaction network between pathways. The overall and systematical pathway analysis of the relationship between marked pathways can help to disclose the synergistic effect module of important pathways. In the top 10 altered pathway interaction nets with 111 nodes and 404 relationships between each other, MAPK signaling pathway was the most important one with the largest degree (outdegree $=5$, indegree $=39$, and degree $=$ 44) (Table 3) and it was in the centre of the altered pathways interaction network (Figure 3).
3.5. Systematic Analysis of DEGs and Altered Pathway in Different Section and Time Points by SAM and KEGG. The DEG Ccl3 and MAPK signaling pathways are not necessarily in the top 10 list (Tables 4 and 5) because of the difference of data analysis. We only discussed, verified, and confirmed the correlation between DEGs Ccl3, MAPK signaling pathway, and SCI induced chronic NP because of the great diversity of genes and the huge complexity of the whole work. However, what is the most important is that systematic analysis of DEGs and altered pathway in different section and time point by SAM and KEGG suggests another method and strategy to study the target gene and pathway of nerve-related disease.

\section{Discussion}

SCI has been demonstrated to be a polygenic disease and its pathogenic mechanism is associated with changes of many genes. In this study, we have used microarray technology to identify differentially expressed genes (DEGs) and activated signaling pathways in association with SCI induced chronic NP in a mouse SCI model. We showed that $\mathrm{Ccl} 3$ and MAPK were the most upregulated DEG and the most activated signaling pathway, respectively. Our results are consistent 
TABLE 2: Top 10 GO terms and KEGG pathways enrichment results of DEGs. Significantly enriched GO terms and KEGG pathways with FDR $<0.05$ were screened out. KEGG biological pathway enrichment analysis found that MAPK signaling pathway (enrichment score $=5.68, P=$ $3.38 E-74$, and $\mathrm{FDR}=8.78 E-72$ ) was the most important one among the 209 pathways according to the enrichment score.

\begin{tabular}{|c|c|c|c|c|c|}
\hline Pathway ID & Pathway name & Enrichment score & $P$ value & FDR & Rank \\
\hline 4010 & MAPK signaling pathway & 5.68 & $3.38 E-74$ & $8.78 E-72$ & 1 \\
\hline 1100 & Metabolic pathways & 2.73 & $3.22 E-65$ & $4.19 E-63$ & 2 \\
\hline 4151 & PI3K-Akt signaling pathway & 4.31 & $8.74 E-57$ & $7.57 E-55$ & 3 \\
\hline 5200 & Pathways in cancer & 4.36 & $2.57 E-53$ & $1.67 E-51$ & 4 \\
\hline 4380 & Osteoclast differentiation & 6.77 & $2.20 E-52$ & $1.14 E-50$ & 5 \\
\hline 5166 & HTLV-I infection & 4.54 & $6.12 E-52$ & $2.65 E-50$ & 6 \\
\hline 4810 & Regulation of actin cytoskeleton & 5.06 & $3.43 E-49$ & $1.28 E-47$ & 7 \\
\hline 4062 & Chemokine signaling pathway & 5.26 & $6.63 E-49$ & $2.15 E-47$ & 8 \\
\hline 4510 & Focal adhesion & 5.11 & $2.31 E-47$ & $6.68 E-46$ & 9 \\
\hline 5205 & Proteoglycans in cancer & 4.73 & $3.22 E-45$ & $8.36 E-44$ & 10 \\
\hline
\end{tabular}

TABLE 3: The top 10 altered pathways of network analyses. The outdegree and indegree represent, respectively, the number of upstream and downstream signal pathways. The degree represents the sum of the outdegree and indegree. In the top 10 altered pathway interaction nets with 111 nodes and 404 relationships between each other, MAPK signaling pathway was the most important one with the largest degree (outdegree $=5$, indegree $=39$, and degree $=44$ ).

\begin{tabular}{|c|c|c|c|c|c|}
\hline Pathway ID & Pathway name & Outdegree & Indegree & Degree & Rank \\
\hline 4010 & MAPK signaling pathway & 5 & 39 & 44 & 1 \\
\hline 4210 & Apoptosis & 3 & 29 & 32 & 2 \\
\hline 5200 & Pathways in cancer & 28 & 0 & 28 & 3 \\
\hline 4110 & Cell cycle & 3 & 20 & 23 & 4 \\
\hline 10 & Glycolysis/gluconeogenesis & 5 & 15 & 20 & 5 \\
\hline 4020 & Calcium signaling pathway & 5 & 14 & 19 & 6 \\
\hline 4115 & p53 signaling pathway & 2 & 17 & 19 & 7 \\
\hline 4310 & Wnt signaling pathway & 8 & 9 & 17 & 8 \\
\hline 4060 & Cytokine-cytokine receptor interaction & 0 & 16 & 16 & 9 \\
\hline 620 & Pyruvate metabolism & 7 & 8 & 15 & 10 \\
\hline
\end{tabular}

with that of previous studies. It will be very interesting to further this study into SCI patients. Throughout the analysis, the factors affecting the results include sample attributes (sample source, sample size, and sample quality), treatment tools, treatment methods, and results screening. In addition, various analysis methods were used, including the screening of differentially expressed genes, KEGG pathway enrichment analyses, and pathway network analyses. All the analysis processes were performed on the GCBI platform in order to avoid the error difference resulting from running different analysis at the different platforms.

The activation of resident cells and the inflammatory cells (macrophages, neutrophils, and lymphocytes) in PNS was involved in peripheral sensitization. In the spinal dorsal horn, glial cells (microglia and astrocytes) are activated to account for central sensitization. Neuropathic pain induced by peripheral and central sensitization is mediated by some inflammatory mediators (IFMs) including chemokines and cytokines (e.g., Ccl3) [39]. After SCI, Ccl3 were induced significantly in the dorsal horns 2 days after lesion and remained at high levels with significantly higher intensities

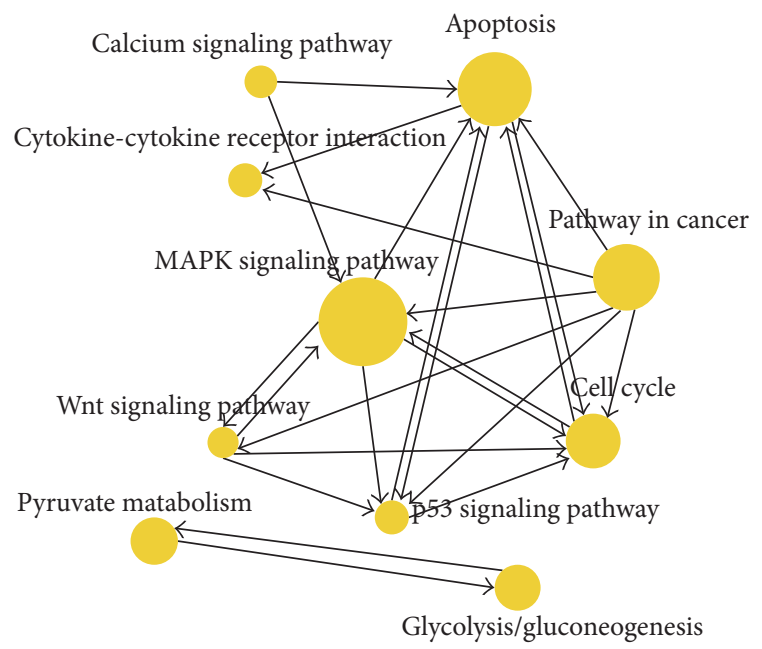

Figure 3: Pathway network after spinal cord injury. The more important the signaling pathway is, the larger the ball is. The importance was ranked according to the degree. MAPK signaling pathway was in the centre of the altered pathways interaction net. 
TABLE 4: Top 10 DEGs and pathways between sham-injury and injury in lesion centre at different time points. At different time points, top 10 DEGs and pathways between sham-injury and injury in lesion centre are showed, respectively.

\begin{tabular}{|c|c|c|}
\hline Time point & $\begin{array}{l}\text { Top } 10 \text { DEGs between } \\
\text { sham-injury and injury in lesion } \\
\text { centre }\end{array}$ & Top 10 pathways between sham-injury and injury in lesion centre \\
\hline $0.5 \mathrm{~h}$ & $\begin{array}{l}\text { Npas4, Gm2083, Socs3, Socs3, } \\
\text { Fosb, Ccl3, II6, Cyr61, Ptgs2, } \\
\text { Myh1 }\end{array}$ & $\begin{array}{l}\text { Pathways in cancer, MAPK signaling pathway, Transcriptional misregulation in } \\
\text { cancer, focal adhesion, proteoglycans in cancer, PI3K-Akt signaling pathway, hippo } \\
\text { signaling pathway, HTLV-I infection, regulation of actin cytoskeleton, metabolic } \\
\text { pathways }\end{array}$ \\
\hline $4 \mathrm{~h}$ & $\begin{array}{l}\text { Ucn2, Gm2083, Atf3, Hspalb, } \\
\text { Hspalb, Ccl3, C330006P03Rik, } \\
\text { Hspala, Hspalb, Egr3 }\end{array}$ & $\begin{array}{c}\text { Metabolic pathways, MAPK signaling pathway, pathways in cancer, PI3K-Akt } \\
\text { signaling pathway, HTLV-I infection, focal adhesion, proteoglycans in cancer, } \\
\text { osteoclast differentiation, transcriptional misregulation in cancer, olfactory } \\
\text { transduction }\end{array}$ \\
\hline $24 \mathrm{~h}$ & $\begin{array}{l}\text { Gm2083, Socs3, Chi313, Adam8, } \\
\text { Gp49a, Hmoxl, Serpinel, Tgm1, } \\
\text { A130040M12Rik, Tnc }\end{array}$ & $\begin{array}{l}\text { Metabolic pathways, MAPK signaling pathway, pathways in cancer, HTLV-I } \\
\text { infection, PI3K-Akt signaling pathway, focal adhesion, protein processing in } \\
\text { endoplasmic reticulum, regulation of actin cytoskeleton, Epstein-Barr virus } \\
\text { infection, proteoglycans in cancer }\end{array}$ \\
\hline $3 \mathrm{~d}$ & $\begin{array}{l}\text { Gpnmb, Cd36, Abcal, Cd51, } \\
\text { Cd36, Ccnb1, Thbs1, Rrm2, } \\
\text { Rrm2, Sprrla }\end{array}$ & $\begin{array}{l}\text { Metabolic pathways, HTLV-I infection, pathways in cancer, focal adhesion, } \\
\text { regulation of actin cytoskeleton, PI3K-Akt signaling pathway, proteoglycans in } \\
\text { cancer, MAPK signaling pathway, lysosome, osteoclast differentiation }\end{array}$ \\
\hline $7 \mathrm{~d}$ & $\begin{array}{l}\text { Gpnmb, Gp49a, Cd36, Cd36, } \\
\text { Ms4a7, Cd5l, C3ar1, Clec7a, } \\
\text { Cd68, Atp6v0d2 }\end{array}$ & $\begin{array}{l}\text { Focal adhesion, PI3K-Akt signaling pathway, metabolic pathways, pathways in } \\
\text { cancer, proteoglycans in cancer, MAPK signaling pathway, regulation of actin } \\
\text { cytoskeleton, osteoclast differentiation, HTLV-I infection, tuberculosis }\end{array}$ \\
\hline $28 \mathrm{~d}$ & $\begin{array}{l}\text { Gpnmb, Clec7a, Cst7, Gp49a, } \\
\text { Lgals3, Cd68, C3ar1, Ms4a7, } \\
\text { Sprrla, Cd48 }\end{array}$ & $\begin{array}{l}\text { Metabolic pathways, MAPK signaling pathway, pathways in cancer, HTLV-I } \\
\text { infection, focal adhesion, proteoglycans in cancer, PI3K-Akt signaling pathway, } \\
\text { regulation of actin cytoskeleton, chemokine signaling pathway, phagosome }\end{array}$ \\
\hline
\end{tabular}

TABLE 5: Top 10 DEGs and pathways between sham-injury and injury in different sections. By processing data from all time points in each section, top 10 DEGs and pathways between sham-injury and injury are showed, respectively.

\begin{tabular}{lcr}
\hline Section & $\begin{array}{c}\text { Top 10 DEGs between } \\
\text { sham-injury and injury }\end{array}$ & Top 10 pathways between sham-injury and injury \\
rostral regions & $\begin{array}{c}\text { Ccl3, Plek, Slc15a3, Bcl2ala, Plek, } \\
\text { Tlr2, Ccl4, Clec7a, Plek, Palld }\end{array}$ & $\begin{array}{c}\text { Osteoclast differentiation, cytokine-cytokine receptor interaction, PI3K-Akt } \\
\text { signaling pathway, phagosome, Chagas disease (American trypanosomiasis), } \\
\text { leishmaniasis, toll-like receptor signaling pathway, chemokine signaling pathway, } \\
\text { tuberculosis, transcriptional misregulation in cancer }\end{array}$ \\
lesion centre & $\begin{array}{c}\text { Ccl3, Atf3, Plek, Ctla2b, Bcl2ala, } \\
\text { Ch25h, Plek, Tnfaip3, Tgifl, } \\
\text { Gpr84 }\end{array}$ & $\begin{array}{c}\text { MAPK signaling pathway, metabolic pathways, PI3K-Akt signaling pathway, } \\
\text { pathways in cancer, osteoclast differentiation, HTLV-I infection, regulation of actin } \\
\text { cytoskeleton, chemokine signaling pathway, Focal adhesion, proteoglycans in cancer }\end{array}$ \\
caudal regions & $\begin{array}{c}\text { Atf3, Tlr2, Irgm1, Trim30d, } \\
\text { Slpr3, Bcl2ala, Slc45a3, Plek, } \\
\text { Trim30a, Zfp36l1 }\end{array}$ & $\begin{array}{c}\text { Tuberculosis, phagosome, Staphylococcus aureus infection, leishmaniasis, osteoclast } \\
\text { differentiation, antigen processing and presentation, herpes simplex infection, Fc } \\
\text { gamma R-mediated phagocytosis, viral myocarditis, Toll-like receptor signaling } \\
\text { pathway }\end{array}$ \\
\hline
\end{tabular}

[40], while, after peripheral nerve injury, $\mathrm{Ccl} 3$ and their receptors (CCR2 and CCR1/CCR5, resp.) were increased [41]. In addition, there were differences in gene expression at the different stages of pain. For example, the expression of $\mathrm{Ccl} 3$ at the six time points was reflected in the ranking of top 10 DEGs (Table 5). Because of the existence of ongoing pain and evoked pain following SCI, here the neuropathic pain we discussed is defined as the evoked pain following SCI. Moreover, we did not perform animal experiments to confirm the relationship between the protein function and SCI-NP, and we did not exclude the false positive microarray results, which result from insufficient conditions.

Ccl3, the ligand of CCR1 [42] and CCR5 [43, 44], was upregulated after SCI and elicits chronic inflammation, resulting in NP $[45,46]$. Peripheral Ccl3 $[47,48]$ and $\mathrm{Ccl} 3$ in the spinal cord $[49,50]$ can produce pain behaviors through the activation of chemokine receptors in the dorsal root ganglia (DRG). Ccl3 was found to be upregulated in activated Schwann cells and infiltrating macrophages close to the injured nerves and found to participate in the development of neuropathic pain through its dominant receptors CCR1 and CCR5, which are also located in Schwann cells and macrophages [39].

CCR1 were found to be induced in the early phase (first 7 days after SCI), while in the late time course (42 days after SCI) elevated chemokine levels were only found after severe SCI [42]. CCR5 was involved in the development of other inflammatory diseases through macrophage activation 
[51-53], which was located in primary afferent neurons or secondary neurons of the spinal dorsal horn $[47,54]$. $\mathrm{Ccl} 3$ and its receptor, CCR5, are upregulated in the spinal cord after injury by using qRT-PCR analysis $[43,44]$.

Microglia and astrocytes constitutively express CCR1 and CCR5 $[55,56]$. It has been shown that microglia proliferate robustly after SCI and were essential to induce NP sensitization [57, 58]. Furthermore, minocycline, a microglial inhibitor, was reported to prevent, delay, or relieve NP [59, $60]$. On the contrary, microglial activation is sufficient to induce pain sensitization [61]. Microglia are referred to as a main source of IFMs in the CNS $[62,63]$, which plays a crucial role in neuropathic pain development [64].

MAPK signaling pathway and chemokine signaling pathway are involved in SCI, which play a very important role in SCI induced NP [65]. MAPK family includes three major members: p38, extracellular signal regulated kinase (ERK), and c-Jun $\mathrm{N}$-terminal kinase (JNK), regulating different signaling pathways. MAPKs are activated by phosphorylation and transduce a broad range of extracellular stimuli by both transcriptional and nontranscriptional regulation, leading to different intracellular responses. Asiaticoside attenuates SCI induced NP through anti-inflammatory effects and inhibition of the p38-MAPK mechanism [66]. Intrathecal injection of the anti-inflammatory cytokine alleviated SCI induced inflammation, suppressing the SCI induced activation of p38-MAPK [67]. Moreover, CCR5 is one receptor of Ccl3, knockout of CCR5 suppressed SCI induced neuropathic pain [67]. Inhibition of p38 MAPK signaling pathway can alleviate neuropathic pain [68].

p38 MAPK is activated by upstream kinase MKK3/MMK6, whose activation in spinal cord microglia was reported after SCI model [69]. p38 $\alpha$ and p38 $\beta$ are two major p38 isoforms among the four isoforms: $\alpha, \beta, \gamma$, and $\delta$ in the mature nervous system [70]. p38 $\beta$ appears to be expressed in spinal cord microglia, and the knockdown of $\mathrm{p} 38 \beta$ but not $\mathrm{p} 38 \alpha$ prevents acute pain sensitization [71]. p38 is involved in the maintenance of neuropathic pain, and its inhibitor can attenuate and reverse NP symptoms [57].

Activation of cytokine receptors (CCR1 and CCR5) results in p38 MAPK activation in spinal cord microglia. p38 activation results in increased expression, through the transcription factor $\mathrm{NF}-\kappa \mathrm{B}$ or other transcription factors (e.g., ATF-2), of secreted inflammatory mediators/growth factors (e.g., cytokines and BDNF) or of genes encoding membrane receptors. In addition, p38 also induces release of PGE 2 and IL- $1 \beta$ via rapid posttranslational regulation. Upon release, these mediators will sensitize nociceptive dorsal horn neurons via presynaptic and postsynaptic mechanisms, leading to persistent pain hypersensitivity [57].

Furthermore, exosomes and other extracellular vesicles are emerging as a novel form of information exchange within the nervous system, and exosomes can play both neuroprotective and neurotoxic roles [72]. Exosomes are released by neurons in a way depending on synaptic activity, and these exosomes can be retaken by other neurons, suggesting a novel way for interneuronal communication [73]. Exosomes derived from heat-stressed tumor cells (HS-TEX) which contain chemokines, such as CCL2, CCL3, CCL4,
CCL5, and CCL20, could chemoattract and activate dendritic cells (DC) and T cells more potently [74]. Schwann cellsderived exosomes enhance axonal regeneration and increase neuronal survival after prodegenerative stimulation [75]. The cotransplantation of Schwann cells and OECs reduced number of astrocytes, microglia and macrophage infiltration, and the expression of chemokines (CCL2 and CCL3) at the injured site, which provide a better immune environment for SCI repair [76].

$\mathrm{Ccl} 3$ and its receptors, CCR5 and CCR1, are upregulated after SCI, and knockout of $\mathrm{Ccl} 3$ as well as inhibition of p38 MAPK signaling pathway can alleviate neuropathic pain [67]. Thus, $\mathrm{Ccl} 3$ antagonists may be potential new drugs for the treatment of neuropathic pain.

\section{Conclusions}

In this study, the maximum change of gene expression profile $\mathrm{Ccl} 3$ (fold change $=10.91, P=2.20 E-05$ ) was identified among the altered 529 DEGs after SCI with threshold of $P<0.05$ and fold change $>2$. Furthermore, KEGG analysis found that 209 pathways with significance were identified, among which the most important was the MAPK signaling pathway according to the enrichment score (enrichment score $=5.68, P=3.38 E-74$, and Fold Discovery Rate $(\mathrm{FDR})=8.78 E-72)$. According to previous study, in SCI induced chronic NP, exosomes in the peripheral blood would contain $\mathrm{Ccl} 3$, which was derived from Schwann cells. The exosomes could cross blood-spinal cord barrier and combine with Ccl3's receptor, CCR5, which accounts for the chronic neuropathic pain syndromes. $\mathrm{Ccl} 3$ and its receptor, CCR5, are also upregulated after SCI, and knockout of $\mathrm{Ccl} 3$ as well as inhibition of p38 MAPK signaling pathway can alleviate neuropathic pain. Since the exosomes with $\mathrm{Ccl} 3$ can be easily and efficiently detected in peripheral blood, $\mathrm{Ccl} 3$ may serve as a potentially prognostic and predictive target for the diagnosis and treatment of SCI induced chronic NP in clinical applications. What is the most, the systematic analysis of DEGs and altered pathway in different section and time point by SAM and KEGG suggests another method and strategy to study the target gene and pathway of nerve-related disease.

\section{Conflicts of Interest}

All authors declare no conflicts of interest.

\section{Acknowledgments}

This work was supported by grants from the National Natural Science Foundation of China (NSFC no. 81171719) and from Chongqing Natural Science of Foundation of China (CSTC, 2008 BB5281). The authors thank Dr. Dongsheng Wu for reading and revising this manuscript.

\section{References}

[1] J. Margolis, P. Juneau, A. Sadosky, J. C. Cappelleri, T. Bryce, and E. Nieshoff, "Health care utilization and expenditures among Medicaid beneficiaries with neuropathic pain following spinal cord injury," Journal of Pain Research, vol. 7, pp. 379-387, 2014. 
[2] P. J. Siddall, J. M. McClelland, S. B. Rutkowski, and M. J. Cousins, "A longitudinal study of the prevalence and characteristics of pain in the first 5 years following spinal cord injury," Pain, vol. 103, no. 3, pp. 249-257, 2003.

[3] C. J. Vierck, P. Siddall, and R. P. Yezierski, "Pain following spinal cord injury: animal models and mechanistic studies," Pain, vol. 89, no. 1, pp. 1-5, 2000.

[4] J. Gómez-Soriano, E. Goiriena, J. Florensa-Vila et al., "Sensory function after cavernous haemangioma: a case report of thermal hypersensitivity at and below an incomplete spinal cord injury," Spinal Cord, vol. 50, no. 9, pp. 711-715, 2012.

[5] N. B. Finnerup, I. L. Johannesen, F. W. Bach, and T. S. Jensen, "Sensory function above lesion level in spinal cord injury patients with and without pain," Somatosensory \& Motor Research, vol. 20, no. 1, pp. 71-76, 2009.

[6] N. FInnerup, L. Pedersen, A. Terkelsen, I. Johannesen, and T. Jensen, "Reaction to topical capsaicin in spinal cord injury patients with and without central pain," Experimental Neurology, vol. 205, no. 1, pp. 190-200, 2007.

[7] H. Kumru, D. Soler, J. Vidal, J. M. Tormos, A. Pascual-Leone, and J. Valls-Sole, "Evoked potentials and quantitative thermal testing in spinal cord injury patients with chronic neuropathic pain," Clinical Neurophysiology, vol. 123, no. 3, pp. 598-604, 2012.

[8] T. N. Bryce et al., "Background and description," in Proceedings of the International spinal cord injury pain classification: part I, vol. 50 (6), pp. 413-417, March 6-7, 2009.

[9] N. B. Finnerup, C. Norrbrink, K. Trok et al., "Phenotypes and predictors of pain following traumatic spinal cord injury: a prospective study," The Journal of Pain, vol. 15, no. 1, pp. 40-48, 2014.

[10] G. Zeilig, S. Enosh, D. Rubin-Asher, B. Lehr, and R. Defrin, "The nature and course of sensory changes following spinal cord injury: predictive properties and implications on the mechanism of central pain," Brain, vol. 135, no. 2, pp. 418-430, 2012.

[11] C. Baastrup and N. B. Finnerup, "Pain in spinal cord injury," Pain Management, vol. 2, no. 1, pp. 87-94, 2012.

[12] N. Torrance, B. H. Smith, M. I. Bennett, and A. J. Lee, "The epidemiology of chronic pain of predominantly neuropathic origin. Results from a general population survey," The Journal of Pain, vol. 7, no. 4, pp. 281-289, 2006.

[13] D. Bouhassira, M. Lantéri-Minet, N. Attal, B. Laurent, and C. Touboul, "Prevalence of chronic pain with neuropathic characteristics in the general population," Pain, vol. 136, no. 3 , pp. 380-387, 2008.

[14] H. Klit, N. B. Finnerup, and T. S. Jensen, "Central post-stroke pain: clinical characteristics, pathophysiology, and management," The Lancet Neurology, vol. 8, no. 9, pp. 857-868, 2009.

[15] N. B. Finnerup and C. Baastrup, "Spinal cord injury pain: mechanisms and management," Current Pain and Headache Reports, vol. 16, no. 3, pp. 207-216, 2012.

[16] P. L. Foley, H. M. Vesterinen, B. J. Laird et al., "Prevalence and natural history of pain in adults with multiple sclerosis: systematic review and meta-analysis," Pain, vol. 154, no. 5, pp. 632-642, 2013.

[17] J. Xu, X. E, H. Liu et al., "Tumor necrosis factor-alpha is a potential diagnostic biomarker for chronic neuropathic pain after spinal cord injury," Neuroscience Letters, vol. 595, pp. 3034, 2015.
[18] P. W. New and R. Marshall, "International spinal cord injury data sets for non-traumatic spinal cord injury," Spinal Cord, vol. 52, no. 2, pp. 123-132, 2014.

[19] F. Biering-Sorensen, A. Bryden, A. Curt et al., "International spinal cord injury upper extremity basic data set version 1.1," Spinal Cord, vol. 53, no. 12, pp. 890-890, 2015.

[20] H. Sabelström, M. Stenudd, and J. Frisén, "Neural stem cells in the adult spinal cord," Experimental Neurology, vol. 260, no. 6, pp. 44-49, 2014.

[21] C. Chen, F. Chen, C. Yao et al., "Intrathecal Injection of Human Umbilical Cord-Derived Mesenchymal Stem Cells Ameliorates Neuropathic Pain in Rats," Neurochemical Research, vol. 41, no. 12, pp. 3250-3260, 2016.

[22] N. Seyedhassantehrani, Y. Li, and L. Yao, "Dynamic behaviors of astrocytes in chemically modified fibrin and collagen hydrogels," Integr. Biol., vol. 8, no. 5, pp. 624-634, 2016.

[23] C. Nicaise, "Transplantation of stem cell-derived astrocytes for the treatment of amyotrophic lateral sclerosis and spinal cord injury," World Journal of Stem Cells, vol. 7, no. 2, p. 380, 2015.

[24] T. Chu, H. Zhou, F. Li, T. Wang, L. Lu, and S. Feng, "Astrocyte transplantation for spinal cord injury: Current status and perspective," Brain Research Bulletin, vol. 107, pp. 18-30, 2014.

[25] M. Noble, J. E. Davies, M. Mayer-Pröschel, C. Pröschel, and S. J. Davies, "Precursor Cell Biology and the Development of Astrocyte Transplantation Therapies: Lessons from Spinal Cord Injury," Neurotherapeutics, vol. 8, no. 4, pp. 677-693, 2011.

[26] Z. Cheng, W. Zhu, K. Cao et al., "Anti-Inflammatory Mechanism of Neural Stem Cell Transplantation in Spinal Cord Injury," International Journal of Molecular Sciences, vol. 17, no. 9, p. 1380, 2016.

[27] C. Zhang, F. Tu, J. Zhang, and L. Shen, "E-cadherin-transfected neural stem cells transplantation for spinal cord injury in rats," Journal of Huazhong University of Science and Technology [Medical Sciences], vol. 34, no. 4, pp. 554-558, 2014.

[28] D. B. Allison, X. Cui, G. P. Page, and M. Sabripour, "Microarray data analysis: from disarray to consolidation and consensus," Nature Reviews Genetics, vol. 7, no. 1, pp. 55-65, 2006.

[29] M. Schena, D. Shalon, R. W. Davis, and P. O. Brown, "Quantitative monitoring of gene expression patterns with a complementary DNA microarray," Science, vol. 270, no. 5235, pp. 467-470, 1995.

[30] M. Schena, D. Shalon, R. Heller, A. Chai, P. O. Brown, and R. W. Davis, "Parallel human genome analysis: microarraybased expression monitoring of 1000 genes.", Proceedings of the National Academy of Sciences, vol. 93, no. 20, pp. 10614-10619, 1996.

[31] G. Chen, X. Fang, and M. Yu, "Regulation of gene expression in rats with spinal cord injury based on microarray data," Molecular Medicine Reports, vol. 12, no. 2, pp. 2465-2472, 2015.

[32] K. Kapur, Y. Xing, Z. Ouyang, and W. Wong, "Exon arrays provide accurate assessments of gene expression," Genome Biology, vol. 8, no. 5, p. R82.

[33] Y. Benjamini and Y. Hochberg, "Controlling the false discovery rate: a practical and powerful approach to multiple testing," Journal of the Royal Statistical Society B: Methodological, vol. 57, no. 1, pp. 289-300, 1995.

[34] L. Gautier, L. Cope, B. M. Bolstad, and R. A. Irizarry, "Affy-analysis of Affymetrix GeneChip data at the probe level," Bioinformatics, vol. 20, no. 3, pp. 307-315, 2004.

[35] V. G. Tusher, R. Tibshirani, and G. Chu, "Significance analysis of microarrays applied to the ionizing radiation response," 
Proceedings of the National Academy of Sciences of the United States of America, vol. 98, no. 9, pp. 5116-5121, 2001.

[36] R dei, G.P., SAM (significance analysis of microarrays). 2008: p. 1755-1755.

[37] J. D. Storey, "A direct approach to false discovery rates," Journal of the Royal Statistical Society. Series B: Statistical Methodology, vol. 64, no. 3, pp. 479-498, 2002.

[38] M. B. Eisen, P. T. Spellman, P. O. Brown, and D. Botstein, "Cluster analysis and display of genome-wide expression patterns," Proceedings of the National Academy of Sciences of the United States of America, vol. 95, no. 25, pp. 14863-14868, 1998.

[39] N. Kiguchi, Y. Kobayashi, and S. Kishioka, "Chemokines and cytokines in neuroinflammation leading to neuropathic pain," Current Opinion in Pharmacology, vol. 12, no. 1, pp. 55-61, 2012.

[40] F. Knerlich-Lukoschus, B. von der Ropp-Brenner, R. Lucius, H. M. Mehdorn, and J. Held-Feindt, "Spatiotemporal CCR1, CCL3(MIP-1 $\alpha$ ), CXCR4, CXCL12(SDF-1 $\alpha$ ) expression patterns in a rat spinal cord injury model of posttraumatic neuropathic pain: laboratory investigation," Journal of Neurosurgery: Spine, vol. 14, no. 5, pp. 583-597, 2011.

[41] N. Kiguchi, Y. Kobayashi, F. Saika, and S. Kishioka, "Epigenetic upregulation of CCL2 and CCL3 via histone modifications in infiltrating macrophages after peripheral nerve injury," Cytokine, vol. 64, no. 3, pp. 666-672, 2013.

[42] F. Knerlich-Lukoschus and J. Held-Feindt, "Chemokine-ligands/ receptors: multiplayers in traumatic spinal cord injury," Mediators of Inflammation, vol. 2015, Article ID 486758, 9 pages, 2015.

[43] K. Kwiatkowski, A. Piotrowska, E. Rojewska et al., "Beneficial properties of maraviroc on neuropathic pain development and opioid effectiveness in rats," Progress in Neuro-Psychopharmacology and Biological Psychiatry, vol. 64, pp. 68-78, 2016.

[44] K. Matsushita, H. Tozaki-Saitoh, C. Kojima et al., "Chemokine (C-C motif) Receptor 5 Is an Important Pathological Regulator in the Development and Maintenance of Neuropathic Pain," Anesthesiology, vol. 120, no. 6, pp. 1491-1503, 2014.

[45] S. Shamash, F. Reichert, and S. Rotshenker, "The cytokine network of Wallerian degeneration: tumor necrosis factoralpha, interleukin-1alpha, and interleukin-1beta," J. Neurosci, vol. 22, no. 8, pp. 3052-60-3060, 2002.

[46] K. Okamoto, D. P. Martin, J. D. Schmelzer, Y. Mitsui, and P. A. Low, "Pro- and anti-inflammatory cytokine gene expression in rat sciatic nerve chronic constriction injury model of neuropathic pain," Experimental Neurology, vol. 169, no. 2, pp. 386391, 2001.

[47] S. B. Oh, "Chemokines and glycoprotein120 produce pain hypersensitivity by directly exciting primary nociceptive neurons," J Neurosci, vol. 21, no. 14, pp. 5027-35, 2001.

[48] C. Abbadie, J. A. Lindia, A. M. Cumiskey et al., "Impaired neuropathic pain responses in mice lacking the chemokine receptor CCR2," Proceedings of the National Academy of Sciences of the United States of America, vol. 100, no. 13, pp. 7947-7952, 2003.

[49] N. Kiguchi, Y. Kobayashi, T. Maeda, F. Saika, and S. Kishioka, "CC-chemokine MIP- $1 \alpha$ in the spinal cord contributes to nerve injury-induced neuropathic pain," Neuroscience Letters, vol. 484, no. 1, pp. 17-21, 2010.

[50] Y.-J. Gao, L. Zhang, O. A. Samad et al., "JNK-induced MCP-1 production in spinal cord astrocytes contributes to central sensitization and neuropathic pain," The Journal of Neuroscience, vol. 29, no. 13, pp. 4096-4108, 2009.
[51] S. Sorce, R. Myburgh, and K. Krause, "The chemokine receptor CCR5 in the central nervous system," Progress in Neurobiology, vol. 93, no. 2, pp. 297-311, 2011.

[52] G. Lazennec and A. Richmond, "Chemokines and chemokine receptors: new insights into cancer-related inflammation," Trends in Molecular Medicine, vol. 16, no. 3, pp. 133-144, 2010.

[53] K. L. Jones, J. J. Maguire, and A. P. Davenport, "Chemokine receptor CCR5: from AIDS to atherosclerosis," British Journal of Pharmacology, vol. 162, no. 7, pp. 1453-1469, 2011.

[54] C. Abbadie, "Chemokines, chemokine receptors and pain," Trends in Immunology, vol. 26, no. 10, pp. 529-534, 2005.

[55] M. E. Dorf, M. A. Berman, S. Tanabe, M. Heesen, and Y. Luo, "Astrocytes express functional chemokine receptors," Journal of Neuroimmunology, vol. 111, no. 1-2, pp. 109-121, 2000.

[56] E. W. Boddeke, "Cultured rat microglia express functional betachemokine receptors," Journal of Neuroimmunology, vol. 98, no. 2, pp. 176-84, 1999.

[57] R. R. Ji and M. R. Suter, "p38 MAPK, microglial signaling, and neuropathic pain," Molecular Pain, vol. 3, article 33, 2007.

[58] S. Echeverry, X. Q. Shi, and J. Zhang, "Characterization of cell proliferation in rat spinal cord following peripheral nerve injury and the relationship with neuropathic pain," Pain, vol. 135, no. 1, pp. 37-47, 2008.

[59] V. Raghavendra, F. Tanga, and J. A. Deleo, "Inhibition of microglial activation attenuates the development but not existing hypersensitivity in a rat model of neuropathy," Journal of Pharmacology and Experimental Therapeutics, vol. 306, no. 2, pp. 624-630, 2003.

[60] A. Ledeboer, E. M. Sloane, E. D. Milligan et al., "Minocycline attenuates mechanical allodynia and proinflammatory cytokine expression in rat models of pain facilitation," Pain, vol. 115, no. 1-2, pp. 71-83, 2005.

[61] M. Tsuda, Y. Shigemoto-Mogami, S. Koizumi et al., "P2X4 receptors induced in spinal microglia gate tactile allodynia after nerve injury," Nature, vol. 424, no. 6950, pp. 778-783, 2003.

[62] U. Hanisch, "Microglia as a source and target of cytokines," GLIA, vol. 40, no. 2, pp. 140-155, 2002.

[63] M. Tsuda, K. Inoue, and M. W. Salter, "Neuropathic pain and spinal microglia: a big problem from molecules in "small" glia," Trends in Neurosciences, vol. 28, no. 2, pp. 101-107, 2005.

[64] J. A. M. Coull, S. Beggs, D. Boudreau et al., "BDNF from microglia causes the shift in neuronal anion gradient underlying neuropathic pain," Nature, vol. 438, no. 7070, pp. 1017-1021, 2005.

[65] H. Zhang and Y. Wang, "Identification of molecular pathway changes after spinal cord injury by microarray analysis," Journal of Orthopaedic Surgery and Research, vol. 11, no. 1, 2016.

[66] Y. Luo, C. Fu, Z. Wang, Z. Zhang, H. Wang, and Y. Liu, "Asiaticoside attenuates the effects of spinal cord injury through antioxidant and anti-inflammatory effects, and inhibition of the p38-MAPK mechanism," Molecular Medicine Reports, vol. 12, no. 6, pp. 8294-8300, 2015.

[67] S. Sun, D. Chen, F. Lin et al., "Role of interleukin-4, the chemokine CCL3 and its receptor CCR5 in neuropathic pain," Molecular Immunology, vol. 77, pp. 184-192, 2016.

[68] L. Xia, Y. Zhang, and T. Dong, "Inhibition of MicroRNA-221 Alleviates Neuropathic Pain Through Targeting Suppressor of Cytokine Signaling 1," Journal of Molecular Neuroscience, vol. 59, no. 3, pp. 411-420, 2016.

[69] B. C. Hains and S. G. Waxman, "Activated microglia contribute to the maintenance of chronic pain after spinal cord injury," Journal of Neuroscience, vol. 26, no. 16, pp. 4308-4317, 2006. 
[70] S. Kumar, J. Boehm, and J. C. Lee, "P38 MAP kinases: key signalling molecules as therapeutic targets for inflammatory diseases," Nature Reviews Drug Discovery, vol. 2, no. 9, pp. 717726, 2003.

[71] C. I. Svensson, "Spinal p38beta isoform mediates tissue injuryinduced hyperalgesia and spinal sensitization," J Neurochem, vol. 92, no. 6, pp. 1508-20, 2005.

[72] A. M. Janas, K. Sapoń, T. Janas, M. H. Stowell, and T. Janas, "Exosomes and other extracellular vesicles in neural cells and neurodegenerative diseases," Biochimica et Biophysica Acta (BBA) - Biomembranes, vol. 1858, no. 6, pp. 1139-1151, 2016.

[73] M. Chivet, F. Hemming, K. Pernet-Gallay, S. Fraboulet, and R. Sadoul, "Emerging role of neuronal exosomes in the central nervous system," Frontiers in Physiology, vol. 3, no. 45, pp. 1-6, 2012.

[74] T. Chen, J. Guo, M. Yang, X. Zhu, and X. Cao, "Chemokinecontaining exosomes are released from heat-stressed tumor cells via lipid raft-dependent pathway and act as efficient tumor vaccine," Journal of Immunology, vol. 186, no. 4, pp. 2219-2228, 2011.

[75] R. Lopez-Leal and F. A. Court, "Schwann Cell Exosomes Mediate Neuron-Glia Communication and Enhance Axonal Regeneration," Cellular and Molecular Neurobiology, vol. 36, no. 3, pp. 429-436, 2016.

[76] J. Zhang, H. Chen, Z. Duan et al., "The Effects of Cotransplantation of Olfactory Ensheathing Cells and Schwann Cells on Local Inflammation Environment in the Contused Spinal Cord of Rats," Molecular Neurobiology, vol. 54, no. 2, pp. 943-953, 2017. 

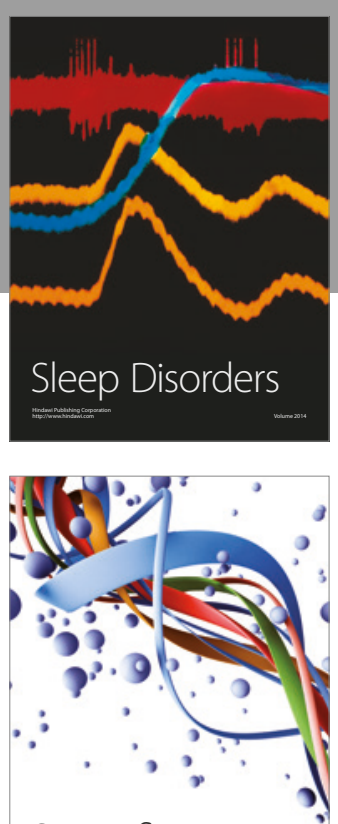

Scientifica
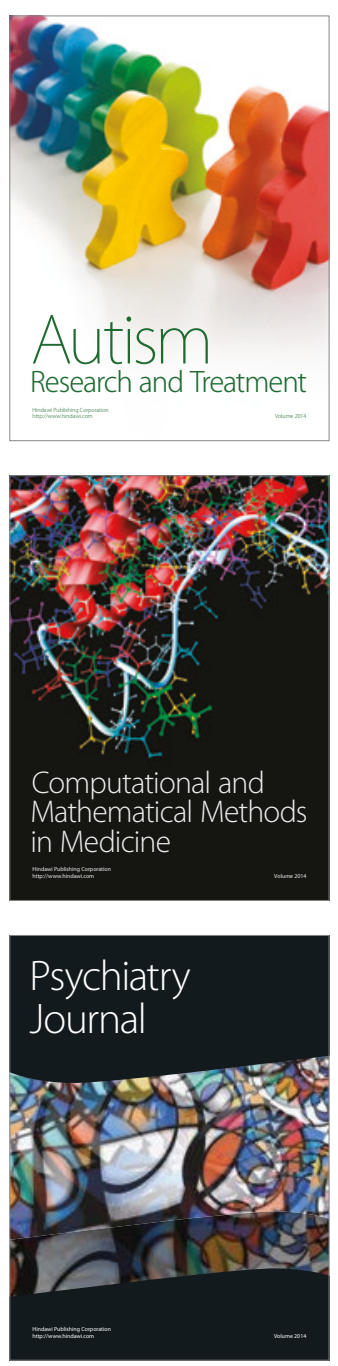
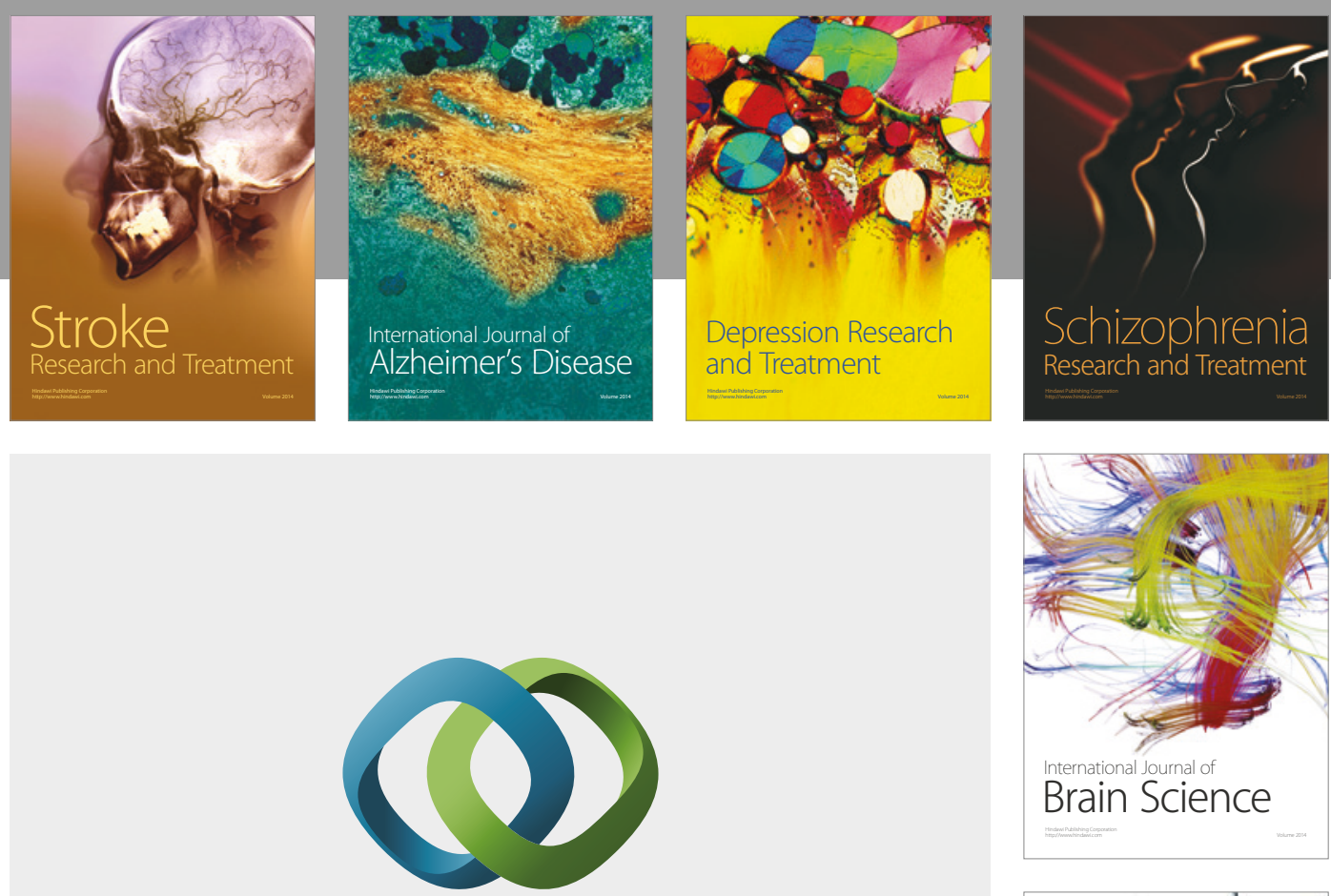

\section{Hindawi}

Submit your manuscripts at

https://www.hindawi.com
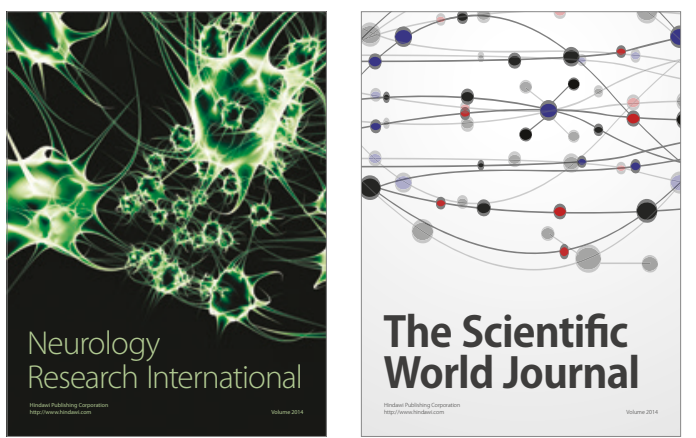

The Scientific World Journal

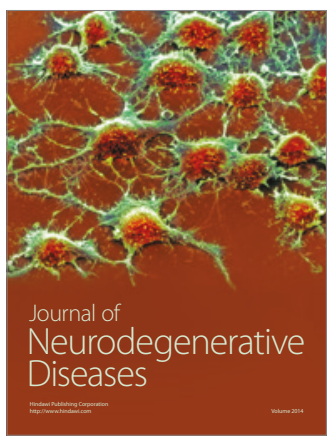

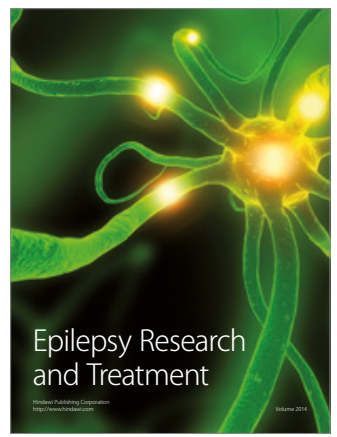

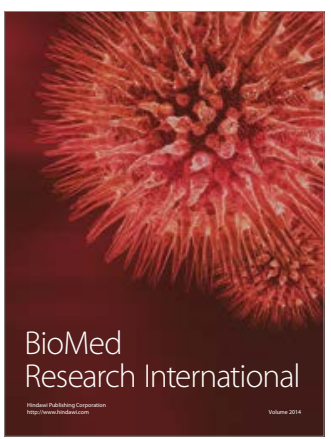

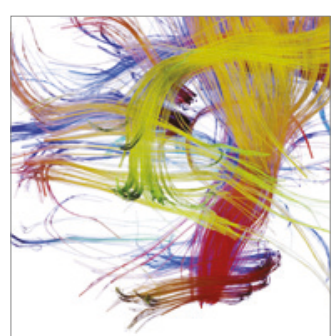

Brain Science

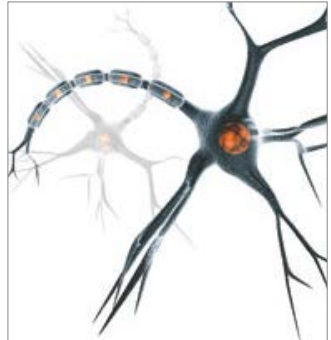

Neural Plasticity
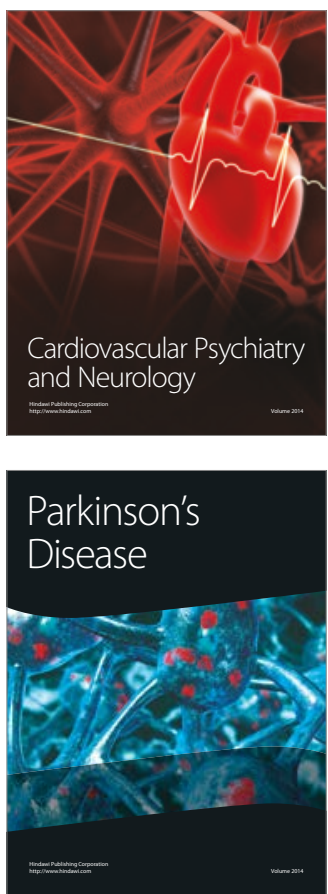